\title{
THE DIFFUSE INTERSTELLAR BANDS IN HISTORY AND IN THE UV
}

\author{
T.P. Snow and J.D. Destree ${ }^{1}$
}

\begin{abstract}
The diffuse interstellar bands (DIBs) have been known of since 1922, but their carrier molecules remain unidentified to this day. We present a brief history of DIB observations, followed by a list of constraints any suggested origin must face, and finally a preview of current research for ultraviolet DIBs using the Cosmic Origins Spectrograph on the Hubble Space Telescope. We conclude that PAHs are consistent with all the listed constraints, but that PAHs may not be the only molecular species responsible for the DIBs.
\end{abstract}

\section{Introduction}

The discovery of the first known diffuse interstellar bands (DIBs) was published in 1922 by Mary Lea Heger, then a graduate student at the Lick Observatory (Heger 1922). After almost a century, the DIBs are now recognized as a main reservoir of organic material in the galaxy - but their origin remains unknown. Some characterize the DIBs as the oldest spectroscopic mystery in all of science. Now, PAHs are among the leading candidate as their carrier molecules.

Recent review papers are Herbig (1995), Snow (2002), Krelowski (2002), and Sarre (2006). An on-line DIB bibliography, listing all published papers on DIBs, can be found at: http://dibdata.org/.

This paper is divided into sections as follows: a brief history of DIB observations; the molecular origin of the DIBs; properties of the DIBs; the possible role of PAHs as carriers of the DIBs; and a report of the first search for UV DIBs done with the Cosmic Origins Spectrograph (COS) recently installed on the Hubble Space Telescope (HST).

\footnotetext{
${ }^{1}$ Center for Astrophysics and Space Astronomy, Department of Astrophysical and Planetary Sciences, University of Colorado at Boulder, Campus Box 389, Boulder, CO 80309-0389, USA
} 


\section{History of DIB observations}

In 1921, while Mary Lea Heger was making observations of "stationary" (i.e. interstellar) atomic sodium absorption lines in stellar spectra, she noted the presence of two broad features at 5780 and $5797 \AA$ that appeared to be interstellar in origin (Heger 1922). Heger took little notice of these features, other than to tabulate them, and it was more than a decade later when Paul Merrill began the first of many systematic studies of the bands.

At about the time when Merrill became actively involved in the problem, several noted astronomers from other fields weighed in with comments on the possible origin of the DIBs (Merrill 1934). Henry Norris Russell, Pol Swings, Meghnad Saha, and Otto Struve, all of them among the fathers of modern astrophysics, commented that the DIBs were likely to have molecular origins. Later, Gerhard Herzberg also became interested in the problem and spent significant effort in attempting to identify possible molecular carriers, though nothing definitive came of this.

In the interval between the 1930s and the 1970s, however, most astronomers interested in the DIB problem favored a non-molecular origin. Instead of gas-phase molecules in the interstellar medium (ISM), dust grains with impurity centers came into vogue. The movement away from molecular explanations was based primarily on the good correlation of DIB strength with interstellar dust extinction, and on perceived improbability of forming and maintaining a significant population of molecules in the diffuse ISM where the DIBs reside. Friedman et al. (2010) summarized the literature on DIB/dust extinction.

An important point to note is that none of the correlations with dust parameters were perfect, implying there is no simple relation between dust and DIBs.

\section{Molecular origins of the DIBs}

One of Herzberg's associates, Alexander E. Douglas, published a very significant paper suggesting that small carbon chain molecules could be the carriers, an idea supported by other authors soon after (Danks \& Lambert 1976; and Smith et al. 1977).

The impetus for the return to the original idea of molecular origins for the DIBs came from several directions: (1) by the 1960s, astronomers had discovered ion-molecule chemistry (long known by then to atmospheric chemists), whose high reaction rates could explain how significant molecular abundances could persist in the interstellar gas; (2) also starting in the 1960s, radio astronomers were beginning to detect rotational emission transitions from interstellar molecules in dark interstellar clouds, lending credence to the general idea that molecules could form and survive in space; (3) in the 1980s strong evidence was found for very large interstellar molecules, specifically the polycyclic aromatic hydrocarbons (PAHs); (4) at about the same time, accurate linear detectors such as CCDs revolutionized observations of the DIBs, allowing for the measurement of very precise band profiles and strengths, far surpassing the older photographic spectra and settling 
a number of issues such as the presence or absence of weak emission wings; and (5) last, but by no means least, in the 1980s scientists from other fields such as chemistry began to take notice of the DIB problem and to lend their expertise to its solution. Chemists are now among the leading users of telescopes for spectroscopic observations of the DIBs, and astronomers have begun to collaborate with chemists in laboratory chemistry experiments on candidate DIB carriers (see the review by Bierbaum et al. in this volume).

In short, starting in the 1980s, the DIB problem has achieved a level of celebrity that it lacked for the first 60 years following Heger's discovery, and has risen to a level of awareness that, we can hope, will lead to a timely resolution.

\section{Properties of the DIBs}

The list below mentions several properties of the DIBs that any successful assignment must explain.

Limited wavelength region: Most are in the red range; almost none are in the blue.

Generally weak strengths: DIBs have very small depths, there are none below 20 percent down from the continuum. Most are less than 5 percent below continuum and less than $30 \mathrm{m \AA}$ in equivalent width.

Specific widths: The broad DIBs are up to $20 \AA$ wide, while the narrow ones are less than 1 or $2 \AA$ wide.

Cloud type dependent: Slightly different sets of DIBs are seen in different environments; they are not constant everywhere. One factor seems to be cloud density, from diffuse to translucent.

Lack of saturation: All DIBs, whether strong or weak, grow linearly with increasing extinction.

Not circumstellar: Several studies of various types of stars with circumstellar material do not show DIBs, despite many searches.

Well-correlated with extinction: All correlations with extinction are good (meaning correlation coefficients are generally above 0.7 ), but they are far from perfect. This includes UV extinction.

Well-correlated with atomic hydrogen: DIBs are significantly better correlated with atomic hydrogen than with molecular hydrogen.

Poor correlations between DIBs: Different authors have defined different sets or "families" of the DIBs that seem to be well correlated. Only one pair of DIBs comes close to being perfectly correlated.

Invariant central wavelengths: DIB central wavelengths show little to no shift between sightlines, except for Doppler shifts with cloud motion. 
Distinct profiles: Many DIBs are asymmetric, though some of the broad ones are symmetric. The profiles are invariant in most lines of sight.

Intrinsic structure: Some narrow DIBs show structure possibly consistent with rotational bands. This is not caused by Doppler shifts, as they are the same everywhere. Broad DIBs generally do not show structure.

Rarely found in emission: DIBs in emission have only been observed in the Red Rectangle and toward one other star.

Lack of emission wings: No DIB has shown emission wings in any environment.

Lack of polarization: Several DIBs have been studied in searches for polarization, with no positive results.

Reasonable elemental composition: Expectations about ISM gas composition should be consistent with the cosmic abundances of common elements.

Lack of UV DIBs: Recent searches have shown that any UV DIBs are weak and do not resemble strong DIBs such as $\lambda 4428$.

\section{PAHs as DIB carriers}

Neutral PAHs tend to have their spectral lines and bands in the UV, which is one reason for thinking ionized PAHs are reasonable candidates for the DIBs carriers (Crawford et al. 1985; Léger \& d'Hendecourt 1985; and van der Zwet \& Allamandola 1985). The neutral/ion ratio depends in the size of the $\mathrm{PAH}-$ the larger the neutral, the greater the tendency to be singly ionized and, thus, to have their spectra in the visible range (Leger \& d'Hendecourt 1985; Bakes \& Tielens 1994; Salama et al. 1996; Le Page et al. 2001, 2003).

It could be that ionized PAHs are responsible for most DIBs because of their better correlation with $\mathrm{H}$ I than with $\mathrm{H}_{2}$ (Fig. 1). In this scenario, the $\mathrm{C}_{2} \mathrm{DIBs}$ (A set of several DIBs that correlate well with $N\left(C_{2}\right) / E(B-V)$, Thorburn et al. 2003) could be located deeper in clouds, where $\mathrm{H}_{2}$ dominates.

The differing physical conditions from cloud to cloud could explain the so-called "families" of DIBs. For example, despite several searches (summarized in Luna et al. 2008), no DIBs have been found in circumstellar environments, where the physical conditions are different from interstellar clouds.

Since PAHs are composed of common elements, the DIB composition requirements are easily met.

The other criteria listed above apply to almost any hypothesis on DIBs origin, except those involving solid-state transitions in dust grains. Note that molecules adhering to dust grains are still reasonably possible DIB carriers. 


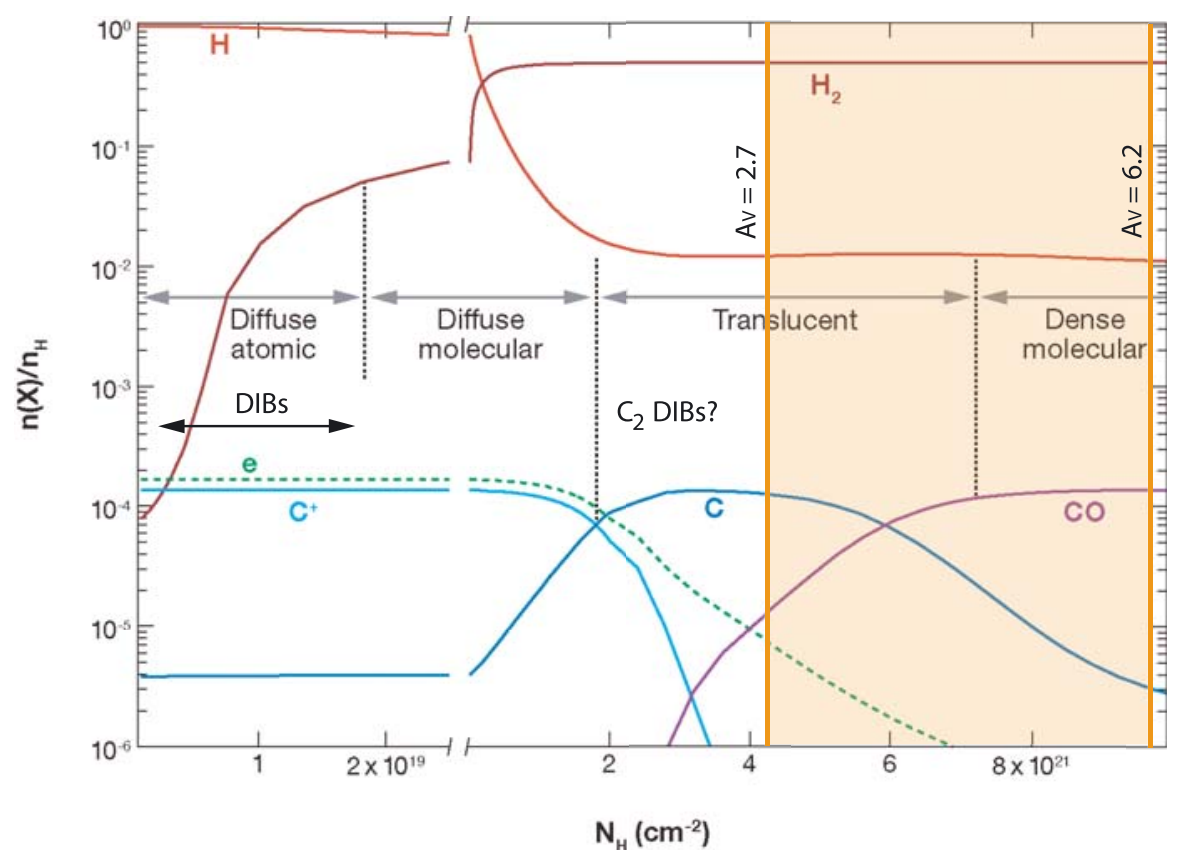

Fig. 1. The physical regimes of the interstellar medium. The ionization levels are shown for hydrogen and carbon, as those species dominate, from diffuse to dense interstellar clouds. The horizontal lines show which types of clouds host the DIBs. The shaded area shows the region now available with the COS.

\section{UV DIBs}

Previous studies have failed to find convincing evidence of DIBs below $3200 \AA$ (Snow et al. 1977; Seab \& Snow 1985). These searches were confined to stars no fainter than about fourth magnitude (Copernicus) to seventh magnitude (International Ultraviolet Explorer). The COS can observe reddened stars to tenth magnitude with $\mathrm{E}_{B-V}$ values of up to 1.5 magnitudes. Hence the $C O S$ has a much better chance of finding UV DIBs that any previous instruments.

Details on the design and performance of the COS can be found in Green et al. (2010, in prep.) and Osterman et al. (2010, in prep.). Figure 2 shows the extinction limits of the COS and several other UV instruments with comparable spectral resolutions. Depending on cloud type as shown by $\mathrm{R}_{V}$ (which denotes the curvature of the extinction curve, in which small values mean high UV extinction and high values mean low UV extinction), we see that the COS is far more sensitive than other instruments in searches for UV DIBs.

We have published one paper on UV DIBs using archival data, finding a few features (Destree \& Snow 2009). These unidentified features are all weak and narrow, nothing comparable to the $4428 \AA$ or $5780 \AA$ features. But we note that broad DIBs were not included in the search, because the HST spectrograph we used 


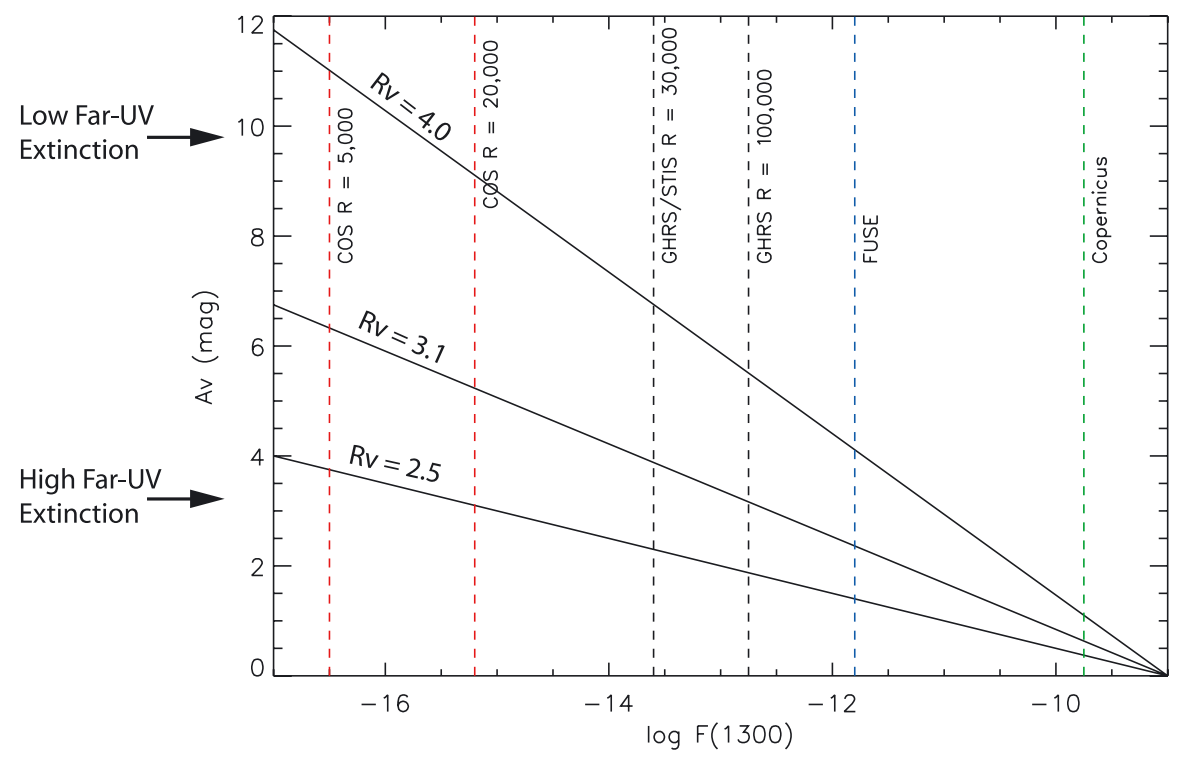

Fig. 2. A comparison of several ultraviolet spectrographs, showing the flux limits (below) and the visual extinction by magnitudes (on the vertical axis). The numbers at the top indicate the spectral resolutions of the various instruments. With low UV extinction (high $\mathrm{R}_{V}$ values), the $C O S$ can observe dense clouds; with high extinction, the COS is more limited, but several magnitudes more sensitive than any previous UV spectrograph.

(the STIS) is an echelle design with many spectral orders covering less than $10 \AA$ each. It is difficult to join orders and match them seamlessly enough to be sure about any broad features. Also, distinguishing between DIBs and instrumental blemishes presents a significant challenge given the current data archive.

The COS has similar problems, though not as severe. In the far UV, meaning approximately $1100 \AA$ to $1700 \AA$, there are two wide spectral bands whose central wavelengths are adjustable. Wide DIBs should be seen, if present. In the near UV ( $1900 \AA$ to $3200 \AA$ ), the spectrograph is not as efficient and does not lend itself to a search for UV DIBs.

In a preliminary search of $C O S$ data we found a few unidentified features, which are apparently weak, but we have more work to do before we can make any definitive conclusions. Unfortunately, we have no observation of unreddened stars of the spectral types to use for comparison, a standard technique for finding visible-wavelength DIBs. Comparison stars or stellar models may be necessary if we want to find very weak or broad UV DIBs.

\section{Final comments}

All the evidence we have so far fits PAHs as the DIB carriers. But PAHs are by no means the only molecular family that might be consistent with the observations. 
It is not necessary to assume that only one family is responsible for the DIBs; it could be a mixture of several different species. We should be careful to have open minds.

This research was supported by NASA grant NNX08AC14G.

\section{References}

Bakes, E.L.O., \& Tielens, A.G.G.M., 1994, ApJ, 427, 822

Crawford, M.K., Tielens, A.G.G.M., \& Allamandola, L.J., 1985, ApJ, 293, L45

Danks, A.J., \& Lambert, D.L., 1976, MNRAS, 174, 57, L25

Douglas, A.E., 1977, Nature, 269, 130

Destree, J.D., \& Snow, T.P., 2009, ApJ, 697, 684 (erratum: ApJ 702, 823)

Friedman et al., 2010, ApJ, submitted

Green, J., et al., 2010, ApJ, in preparation

Heger M.L., 1922, Lick Observatory Bull., 10, 337, 146

Herbig, G.H., 1995, ARA\&A, 33, 19

Herzberg, G., 1955, Mem. R. Soc. Liege, Serie, 15, 326

Krelowski J., 2002, Adv. Space Res., 30, 1395

Leger, A., \& d'Hendecourt, L., 1985, A\&A, 146, 81

Le Page, V., Snow, T.P., \& Bierbaum, V.M., 2001, ApJS, 132, 233

Le Page, V., Snow, T.P., \& Bierbaum, V.M., 2003, ApJ, 584, 316

Luna, R., Cox, N.L.J., Satorre, M.A., et al., 2008, A\&A, 480, 133

Merrill P.W., 1934, PASP, 46, 206

Osterman, S., et al., 2010, ApJ, in preparation

Russell, H.N., 1935, MNRAS, 95, 610

Saha, M.N., 1937, Nature, 139, 840

Salama, F., Bakes, E.L.O., Allamandola, L.J., \& Tielens, A.G.G.M., 1996, ApJ, 458, 621

Sarre, P.J., 2006, J. Molec. Spectr., 238, 1

Sarre, P.J., Miles, J.R., \& Scarrott, S.M., 1995, Science, 269, 674

Seab, C.G., \& Snow, T.P., Jr., 1985, ApJ, 295, 485

Smith, W.H., Snow, T.P., \& York, D.G., 1977, ApJ, 218

Snow, T.P., Jr., York, D.G., \& Resnick, M., 1977, PASP, 89, 758

Snow, T.P., 2002, Spectrochimica Acta Part A, 57, 615

Swings, P., 1937, MNRAS, 97, 212

Thorburn, J.A., et al., 2003, ApJ, 584, 339

van der Zwet, G.P., \& Allamandola, L.J., 1985, A\&A, 146, 76 